\title{
Cluster modeling of the short-range correlation of acoustically emitted scattering signals
}

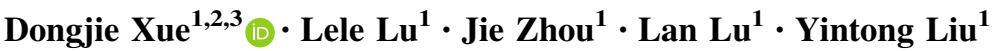

Received: 8 February 2020/Revised: 4 June 2020/Accepted: 30 July 2020/Published online: 20 August 2020

(C) The Author(s) 2020

\begin{abstract}
As a widely used measurement technique in rock mechanics, spatial correlation modeling of acoustic emission (AE) scattering signals is attracting increasing focus for describing mechanical behavior quantitatively. Unlike the statistical description of the spatial distribution of randomly generated AE signals, spatial correlation modeling is based mainly on short-range correlation considering the interrelationship of adjacent signals. As a new idea from percolation models, the covering strategy is used to build the most representative cube cluster, which corresponds to the critical scale at peak stress. Its modeling process of critical cube cluster depends strongly on the full connection of the main fracture network, and the corresponding cube for coverage is termed the critical cube. The criticality pertains to not only the transition of local-to-whole connection of the fracture network but also the increasing-to-decreasing transition of the deviatoric stress with an obvious stress drop in the brittle failure of granite. Determining a reasonable critical cube guarantees the best observation scale for investigating the failure process. Besides, the topological connection induces the geometric criticality of three descriptors, namely anisotropy, pore fraction, and specific surface area, which are evaluated separately and effectively. The results show that cluster modeling based on the critical cube is effective and has criticality in both topology and geometry, as well as the triaxial behavior. Furthermore, the critical cube length presents a high confidence probability of being correlated to the mineral particle size. Besides, its pore fraction of cube cluster is influenced strongly by the critical cube length and confining pressure.
\end{abstract}

Keywords Acoustic emission · Spatial correlation modeling · Cover strategy $\cdot$ Criticality $\cdot$ Critical cube $\cdot$ Cluster modeling

\section{List of symbols}

$d$

$h$

$P$

$\bar{p}$

$q$

$\alpha$

Dongjie Xue

xuedongjie@163.com

1 School of Mechanics and Civil Engineering, China University of Mining and Technology, Beijing 100083, China

2 State Key Laboratory of Coal Mine Disaster Dynamics and Control, Chongqing University, Chongqing 400030, China

3 Key Laboratory of Safety and High-efficiency Coal Mining, Anhui University of Science and Technology, Huainan 232001, China $\beta \quad$ Critical exponent

$f(\alpha) \quad$ Multifractal spectrum

$D(q) \quad$ Generalized fractal dimension

$N_{0}, \delta_{0} \quad$ Initial cube number and cube length

$N, \delta \quad$ Accumulated cube number and any cube length

$p, p_{\mathrm{c}} \quad$ Probability and its critical value

$P_{\mathrm{L}}, \Gamma_{\mathrm{p}} \quad$ Percolation fraction of largest cluster or all clusters

$r_{\text {cyl }}, r_{\mathrm{sph}}$ Cylinder radius and sphere radius

$s_{i}, s_{\mathrm{L}} \quad$ Size of $i$ th acoustic-emission cluster or largest cluster

$\mu, \sigma \quad$ Mean and standard deviation 


\section{Introduction}

When investigating the invisible propagation of fractures in cylindrical rock samples under triaxial compression, acoustic emission (AE) monitoring is commonly used to investigate the brittle or ductile failure of granite (Lei et al. 2000; Chang and Lee 2004; Li and Li 2017) and other hard rocks (Alkan et al. 2007; Yang et al. 2012). By setting a reasonable threshold for voltage, the generated $\mathrm{AE}$ events can be recorded as the spatial coordinates of numerous $\mathrm{AE}$ signals (Hardy 1972; Grosse and Ohtsu 2008). The spatial distribution of location randomness is then analyzed by describing various $\mathrm{AE}$ parameters statistically and conducting harmonic time-series analysis (Hsu et al. 1978; Scruby 1987). However, a remaining challenge is how to establish a quantitative relationship between the triaxial compression behavior and the $\mathrm{AE}$ indicator, and this challenge is due in part to the lack of analysis of the spatial correlation among scattering AE signals.

Usually, the three-dimensional (3D) visualization of randomly distributed $\mathrm{AE}$ signals is done using only scatter plots without spatial correlation analysis. However, the role of the spatial correlation of discrete AE signals cannot be ignored when seeking to illustrate fracture nucleation and evolution. Kaiser (1950) first proposed the memory phenomenon of pre-generated AE signals, termed the Kaiser effect, after which Goodman and Blake (1966) emphasized the spatiotemporal evolution of AE-signal distribution (Stanchits et al. 2015; Meng et al. 2016; Rodríguez et al. 2016). As well as describing the spatial distribution of $\mathrm{AE}$ signals statistically, the enhanced improvement of accuracy in modeling spatiotemporal evolution is influenced strongly by the spatial correlation among AE signals (Hohl et al. 2018). The key to establishing an appropriate association between the triaxial behavior and the associated $\mathrm{AE}$ behavior depends completely on the strategy for modeling of the spatial correlation.

Note that whatever the spatial distribution or spatial correlation of the scattering AE signals, a comprehensive and accurate description of signal cloud is very complex and can be difficult to realize if its geometry and topology are extremely sophisticated. Both fractal theory and percolation theory are important for describing such spatial randomness. Many effective efforts have been made to describe the 3D scatter distribution of AE events (Manthei 2005; Guo et al. 2015; López-Comino et al. 2017), but without analyzing the stochastic interaction and nucleation of fracture network considering the spatial correlation (Lockner 1993). Generally, the fractal description indicates the self-similarity or self-affinity of the AE signal cloud, which is based mainly on the long-range correlation among scattering AE signals (Hirata et al. 1987; Kusunose et al. 1991; Pape et al. 1999; Xie et al. 2011).

However, to describe fracture nucleation, short-range correlation seems more appropriate than long-range correlation among AE signals (Vilhelm et al. 2008; Chevy et al. 2010; Girard et al. 2012), and an extremely important reason for this is that the post-generation of the fracture network is influenced strongly by the pre-existing ones. This dependence exists mainly in a regional 3D cube, not constrained in a simply 1D single-link. Consequently, the local connection among scattering $\mathrm{AE}$ signals exhibits strong short-range correlation. Some achievements referring to the critical nucleation of rock failure (Chelidze 1986; Alkan 2009; Xue et al. 2018) have shown the clear effectiveness of percolation models (Broadbent and Hammersley 1957; Shante and Kirkpatrick 1971; Chelidze 1982; Bebbington et al. 1990; Hunt et al. 2014; Yuan and Bowen 2018) for describing such spatial correlation. Also, percolation models are highly advantageous for describing critical behavior, namely the surge of accumulated $\mathrm{AE}$ events with a stress drop in brittle failure. Considering the short-range correlation, a method known as the cube cluster modeling of scattering $\mathrm{AE}$ signals is proposed herein to describe the spatial correlation.

\section{Materials and methods}

\subsection{Sample preparation}

The granite used in the present study was excavated by drilling at a depth of $550 \mathrm{~m}$ in the Beishan region of Northwestern China, which is an area that has been preselected for the disposal of high-level nuclear waste (Zhao et al. 2013; Wang et al. 2018). It was then processed into cylindrical samples, each with a diameter of $50 \mathrm{~mm}$ and a height of $100 \mathrm{~mm}$.

Beishan granite comprises three main minerals, namely quartz, mica, and feldspar. Rectangular images of the granite surfaces were captured precisely using a high-definition camera, and in those images the particle boundaries were classified based on color variation and calculated using the ImageJ software. The particles were equivalent to circular areas of various sizes, and statistical diameters were determined for each of the three minerals (Fig. 1). The corresponding average diameters of the quartz, mica, and feldspar particles were calculated as $2.26,1.91$, and $2.51 \mathrm{~mm}$, respectively. 

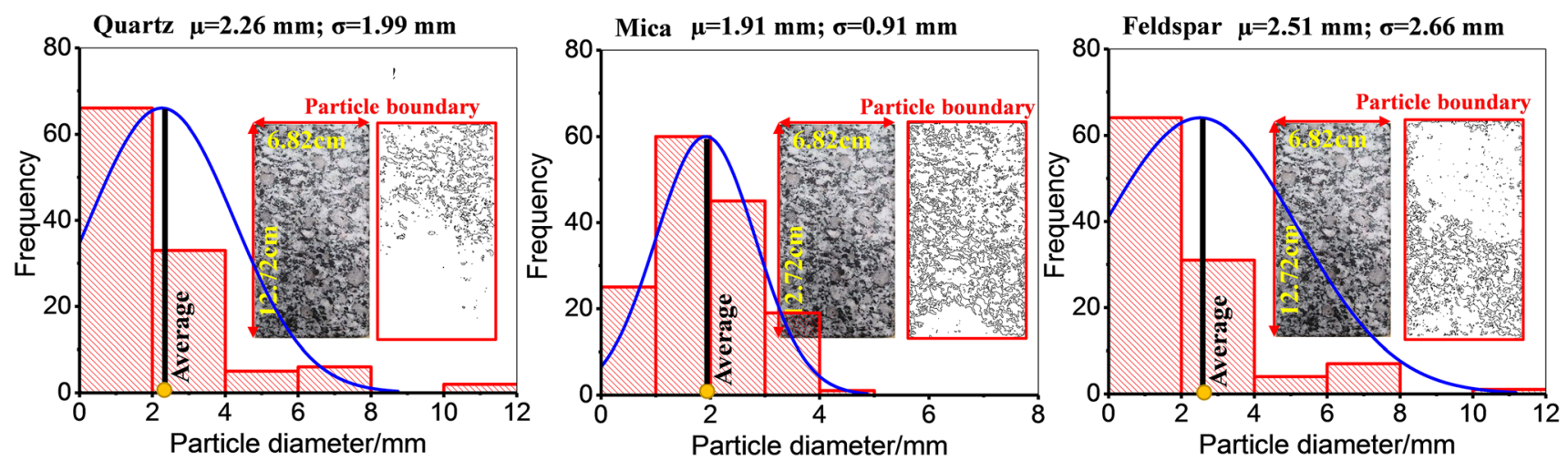

Fig. 1 Normal distributions of particle diameters for the minerals quartz, mica, and feldspar as obtained using the ImageJ software

\subsection{Triaxial compression test and acoustic-emission monitoring}

Triaxial compression was applied using a rock mechanics test system (815 Flex Test GT; MTS, USA), and the AE signals were monitored in real time using an $\mathrm{AE}$ data acquisition and digital signal processing system (PCI-2; Physical Acoustics, USA) and eight AE sensors distributed around the outside of the steel chamber (Fig. 2). Under uniaxial compression, the granite strength was determined as being $161.47 \mathrm{MPa}$, and the Poisson ratio and elastic modulus were 0.15 and $61.20 \mathrm{GPa}$, respectively. Under triaxial compression, the confining pressure was set to $10 \mathrm{MPa}$.

\subsection{Cluster modeling of short-range correlation}

By setting a threshold of $40 \mathrm{~dB}$ for the eight $\mathrm{AE}$ sensors, the $\mathrm{AE}$ signals generated in the cylinder were recorded effectively. Generally, AE signals come from the opening, closing, and friction of random fractures (Tang et al. 2000; Cai et al. 2007; Xue et al. 2020). Besides the spatial coordinates, the memory effect should also include some untapped information about the fracture surface and volume. Further consideration is that before the completeness

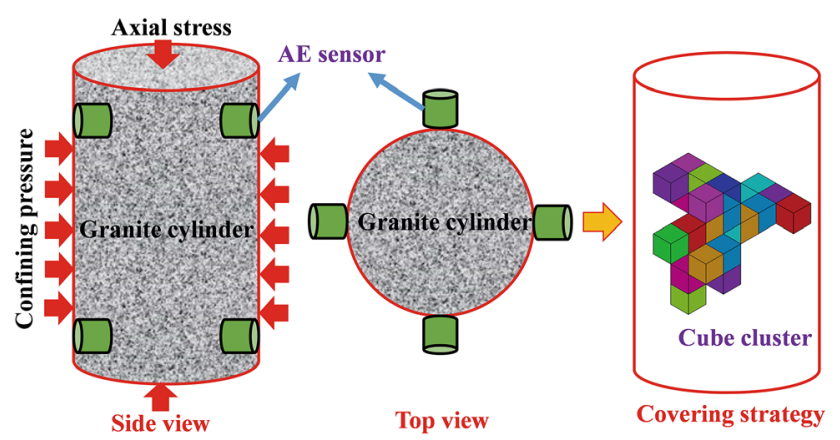

Fig. 2 Schematic of the layout of the eight acoustic emission (AE) sensors for cube covering of the main fracture network, local fractures are the main influences on adjacent zones. Therefore, originating from percolation models, a covering strategy involving various sized 3D cubes could be used to represent the local damaged zone, but the greatest difficulty is how to determine the optimum cube size. The cubes contain all the near-field $\mathrm{AE}$ signals, so the spatial correlations of the $\mathrm{AE}$ scattering signals could be transformed into cube-to-cube connections and then into cluster-to-cluster correlations.

If the covering cubes are too small, with a side length that is much less than the nearest distance between two adjacent points, then all the cubes are isolated, showing a completely discrete state. By contrast, if the cubes are large enough then they contain all the AE signals. A cube containing $\mathrm{AE}$ signals is labeled with a randomely positive number, otherwise it is labeled with zero. Naturally, a zero cube or a cluster of such cubes represents an undamaged part of the matrix. If the cubes are made continuously larger while satisfying the connection criterion, then clusters form containing different cubes, and these various clusters are distinguished and labeled by the increasing number of cube cluster (Fig. 3), which is calculated using

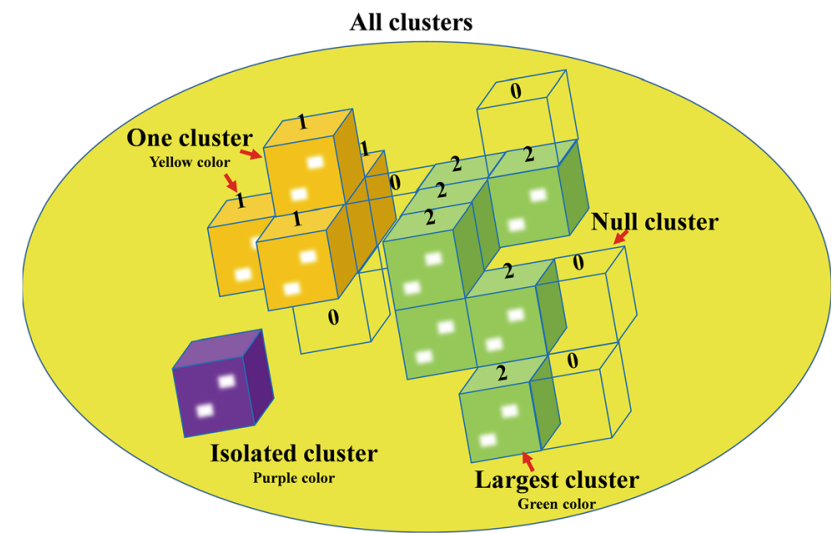

Fig. 3 Schematic of the classification of various cube clusters 
the Hoshen-Kopelman (HK) algorithm (Hoshen et al. 1997; Al-Futaisi and Patzek 2003).

The process of generating cube clusters depends strongly on the connection type. A 3D cube has three geometrical elements, namely points, lines, and surfaces, therefore there are three corresponding connection types, namely point-to-point contact, line-to-line contact, and surface-to-surface contact. Herein, to represent short-range correlations, we consider only surface-to-surface contact (Sugiyama et al. 2010). Different clusters form depending on the size of the cubes that are used, so the crucial issue of the optimum size must be evaluated scientifically, and the cluster corresponding to that size is termed the AE percolation cluster.

\section{Results}

\subsection{Determination of cluster criticality}

Figure 4 shows the accumulated AE evolution with axial strain, as well as a comparison with three other deformable quantities, namely axial strain, hoop strain, and volumetric strain. Obviously, there is a dilatancy-induced surge in the accumulated AE signals from the dilatant stress with the maximum compressive volumetric strain to the peak stress. After the peak stress, there is a rapid drop in stress after brittle failure, which is caused mainly by the full connection of fracture network. Consequently, the evolution of the fracture network (i.e., the connection process) is an important indicator of rock failure.

The suggestion here is that the cube cluster covering the AE signals represents the fracture network. Therefore, the local-to-whole connection must be evaluated precisely. We mark six points on the AE curves to monitor the connection, and in particular the sixth point with the peak stress is selected to describe the transition of the local-to-whole connection, termed the critical connection. The critical connection is therefore an extremely important indicator for evaluating the appropriateness of the covering cubes.

The key to overcoming such a challenge of searching for the critical connection is evaluating the connections accurately and selecting a proper quantitative descriptor. The $\mathrm{HK}$ algorithm is used to predict the connections in real time. If the cubes are too large, then the fracture network is definitely in a state of complete connection; if they are too small, then there are no local connections. There is therefore a critical transition of the local-to-whole connection corresponding to the optimum cube size. This critical connection state has a unique pore fraction that is defined as the volume ratio of the $\mathrm{AE}$ percolation cluster to the cylinder and is taken as the quantitative descriptor.

The pore fraction corresponds uniquely to the cube size. To determine the critical connection, Fig. 5 shows the convergence of calculating the critical cube size by the bisection method to determine critical pore fraction and the abscissa indicates the logarithmic form of cube length, which is normalized and divided by the cylinder diameter.
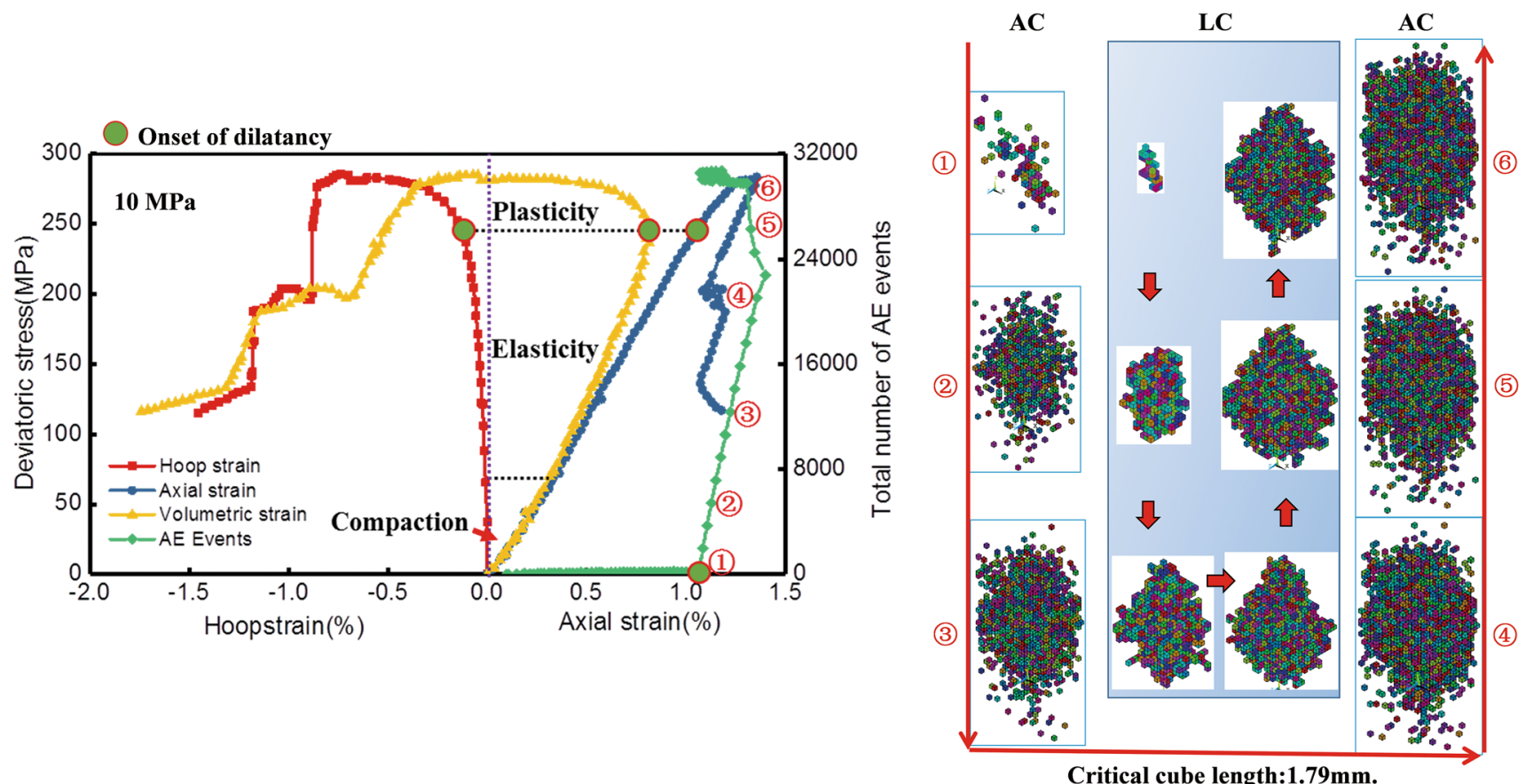

Fig. 4 Complete stress-strain curves referring to the axial, hoop, and volumetric strains as well as the evolution of accumulated AE events and the corresponding cube clusters of the largest cluster (LC) and all clusters (AC) 
(a)
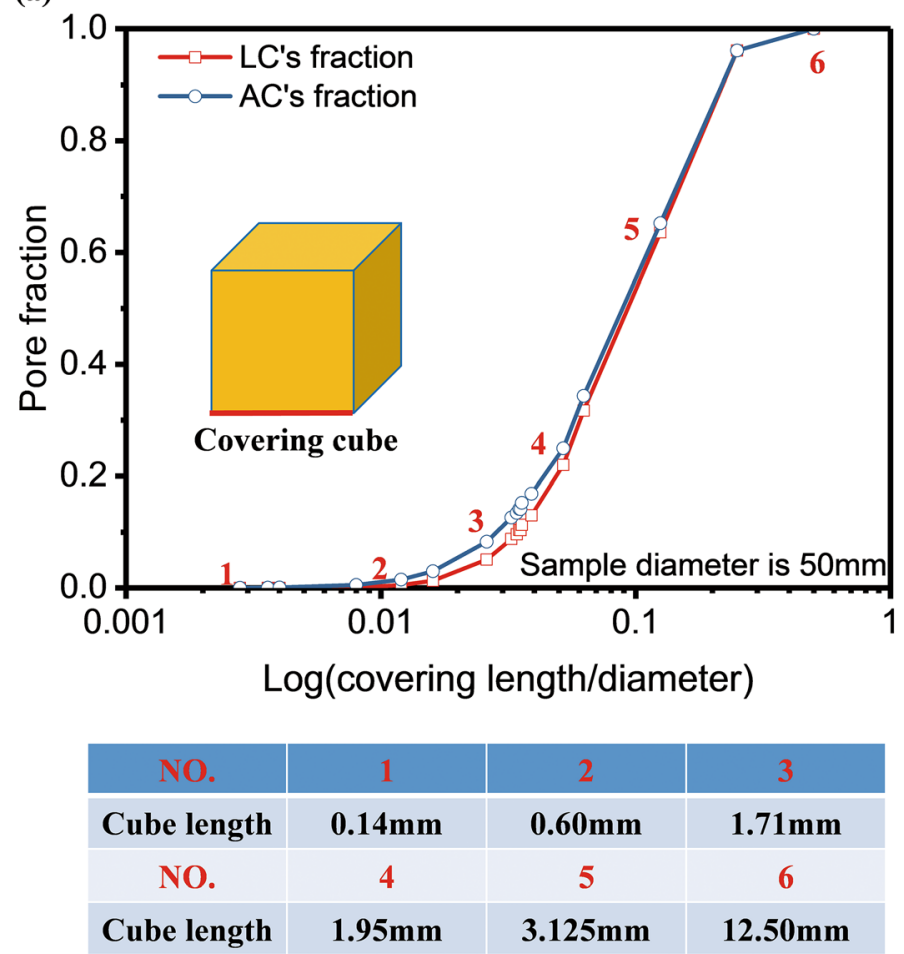

(b)

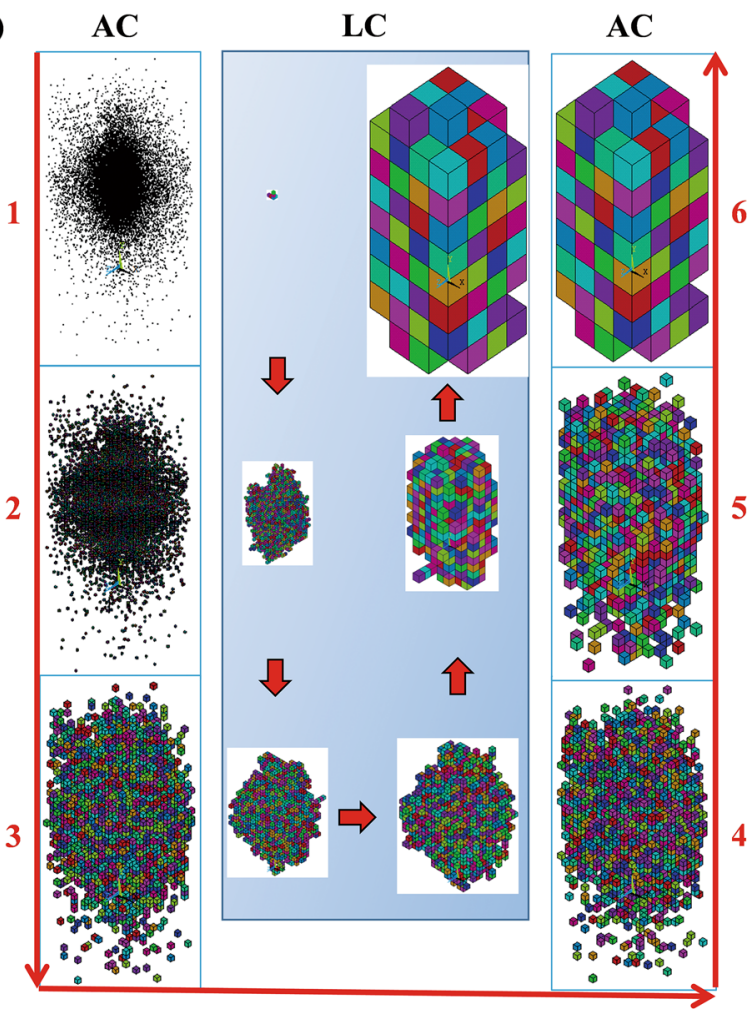

Fig. 5 Determination of critical cube length by a the bisection of pore fraction with the log of cube length divided by cylindrical diameter and b the evolution of multiscale cube clusters for LC and AC

The pore fraction is commonly used to evaluate the similarity between the largest cluster (LC) and all clusters (AC). The curves for LC and AC are similar, indicating a negligible difference, which means that it is reasonable for $\mathrm{LC}$ to represent $\mathrm{AC}$ in the percolation model. Another observation is that the geometric difference between LC and $\mathrm{AC}$ disappears gradually as the covering cubes become larger. Finally, the critical cube length is determined as being $1.79 \mathrm{~mm}$, which is between the third state with a length of $1.71 \mathrm{~mm}$ and the fourth state with a length of $1.95 \mathrm{~mm}$. Figure 1 shows the normal distribution of the particle diameters for each of the three minerals. The two key normal parameters of the mean $\mu$ and standard deviation $\sigma$ are calculated respectively as 2.26 and $1.99 \mathrm{~mm}$ for quartz, 1.91 and $0.91 \mathrm{~mm}$ for mica, and 2.51 and $2.66 \mathrm{~mm}$ for feldspar. The critical cube length is consistent in a confidence interval of $(\mu-\sigma, \mu+\sigma)$ with a probability of $68.27 \%$. That confidence probability shows that the particle scale could be the best means of observing the critical transition of the local-to-whole connection of the fracture network.

\subsection{Cluster criticality regarding anisotropy}

The comfortable cube cluster depends strongly on the criticality of the local-to-whole connection. This transition is also with other critical phenomena of stress drop or surge of cumulative AE signals, so evaluating the comfortability of the cube cluster modeling is to some extent the same as estimating the criticality behavior. In percolation models, there is a classical correlation to describe the critical behavior of

$P \propto\left(p-p_{\mathrm{c}}\right)^{\beta}$

where $P$ is the connectivity probability, $p$ and $p_{\mathrm{c}}$ indicate the causes of critical transition of connectivity and are defined as the probability density of the fracture distribution or some other variable and the critical probability, respectively, and $\beta$ is the critical exponent, which in 3D space equals 2 (Hestir and Long 1990; Aharony and Stauffer 2014; Sakhaee-Pour and Agrawal 2018).

The 3D cube cluster has an extremely complex geometry and topography, as well as complicated connections inside the cluster. Here, the connectivity probability is estimated from the percolation fraction (PF) to illustrate the increasing proportion of LC in AC on different scales. In $\mathrm{AC}$, the PF $\Gamma_{\mathrm{p}}$ indicates the two randomly selected cubes connected in the same cluster and it is defined by (Jarvis et al. 2017)

$\Gamma_{\mathrm{p}}=\frac{\sum_{i} s_{\mathrm{A}}\left(s_{\mathrm{A}}-1\right)}{\sum_{i} s_{i}\left\{\left(\sum_{i} s_{i}\right)-1\right\}} \simeq \frac{\sum_{i} s_{\mathrm{A}}^{2}}{\left(\sum_{i} s_{i}\right)^{2}}$ 
where $s_{\mathrm{A}}$ is the AC size and $i$ is the cube number.

Similarly in LC, the PF $P_{\mathrm{L}}$ is defined by

$P_{\mathrm{L}}=\frac{s_{\mathrm{L}}\left(s_{\mathrm{L}}-1\right)}{\sum_{i} s_{i}\left\{\left(\sum_{i} s_{i}\right)-1\right\}} \simeq \frac{s_{\mathrm{L}}^{2}}{\left(\sum_{i} s_{i}\right)^{2}}$

where $s_{\mathrm{L}}$ is the LC size.

Generally, the complex structure of cube cluster can be reduced to simple geometry to investigate its anisotropy. By the transformation of cube cluster into equivalent geometric volume, the isotropy is often observed by a sphere as well as the anisotropy described by a cylinder. The radius of the equivalent sphere is determined from the cube volume as

$r_{\mathrm{sph}}=\left(\frac{3 N \delta^{3}}{4 \pi}\right)^{1 / 3}$

where $N$ is the total number of covering cubes and $\delta$ is the cube length of side.

Figure 6 shows the nonlinear growth of the sphere's radius with increasing $\mathrm{LC}$ and $\mathrm{AC} \mathrm{PF}$. The nonlinearity is described properly by means of an exponential fit. The increase in the sphere's radius indicates the enhanced distribution of isotropy due to the regional connection of cubes in the physical cylinder. Figure $6 a, b$ show the intersection of two fitted exponential curves for LC and $\mathrm{AC}$, respectively. The point at which the curves cross matches the critical state from the bisection method in Fig. 5. Such a fixed point indicates the stability of the critical cube cluster and the criticality of the transitional behavior of isotropy. The criticality could also be verified further by two lines fitted piecewise; Fig. 7a, b show that the point at which the two lines cross for LC is similar to that for AC. More importantly, the crossing point determined by the two fitted lines corresponds to the fixed point in the exponential description. Therefore, there is an obvious criticality in the isotropy transition from weak to strong. Note that the isotropy here is correlated strongly with the dilatancy, with strong isotropy indicating enhanced dilatancy.

Considering the cylinder shape of the physical sample, its equivalent cylinder is adopted to describe the anisotropy. The two shape parameters that must be determined are the height $h$ and the diameter $r_{\text {cyl }}$. As an important factor, the fracture propagation induces the surge of most of the AE signals. The direction along the line linking two farthest AE points is, with a very high probability, consistent with the fracture propagation. To reveal the anisotropy, the cylinder height is defined as the biggest distance from one $\mathrm{AE}$ point to the farthest one, which is calculated easily by comparing the distances of two randomly selected points. Correspondingly, the bottom radius is determined by
$r_{\mathrm{cyl}}=\sqrt{\left(N \cdot \delta^{3}\right) /(h \cdot \pi)}$

Figure 8a shows the height evolutions for LC and AC. Obviously, the two linear trends with increasing PF are very different. For $\mathrm{AC}$, the height of the equivalent cylinder is nearly constant and very close to the original height of $100 \mathrm{~mm}$. However, for LC the cylinder's height grows linearly with increasing PF, approaching that for AC.

Similar to the isotropy analysis, the fixed point at the intersection of two exponential curves for $\mathrm{LC}$ and $\mathrm{AC}$ (Fig. 8b) also corresponds to the turning point between the two fitting lines (Fig. 8c). The critical transition is also obvious for the anisotropy analysis. With increasing PF, the critical cube cluster is very stable in describing the isotropy or anisotropy. This means that the critical cube dependent on the full connection of the fracture network provides an invariant view of the isotropy and anisotropy.

\subsection{Cluster criticality regarding pore fraction}

Considering the complete damage within the cube cluster, the pore fraction is defined as the volume ratio of the cluster to the physical cylinder. The pore fraction is not equivalent to the fracture porosity, but it does include the locally damaged zones around the fractures. Beyond the damaged cluster, the other part of the granite is undamaged, so the pore fraction here indicates the proportion of damaged volume including the fracture and its adjacent zones.

Figure 9a shows the clear increase in pore fraction with increasing PF for LC and AC. In particular, the increase in pore fraction changes rapidly from slow to fast after the fixed point. The curves fit well to the scatter data while the growth is slow but exhibit serious deviation when the growth is fast. Indeed, the difference in pore fraction between LC and AC is not obvious, with a maximum difference of 0.04 at the boundary corresponding to the critical transition.

Using the linear description of the correlation as shown in Fig. 9b, the transitional boundary can be verified and calculated quantitatively. Correspondingly, the critical PF is determined as 0.70 for both $\mathrm{LC}$ and $\mathrm{AC}$. The two fractions of LC and AC are calculated respectively as 0.09 and 0.13 , which are less than 0.20 , indicate that most of the granite is undamaged. This means that if the fracture connections are observed on the sample scale, then too many undamaged parts will be included, thereby leading to an improper observation scale. Instead, cluster modeling is essential for determining the most appropriate scale. Totally, such transition of pore fraction indicates the criticality of full connection of fracture network. 


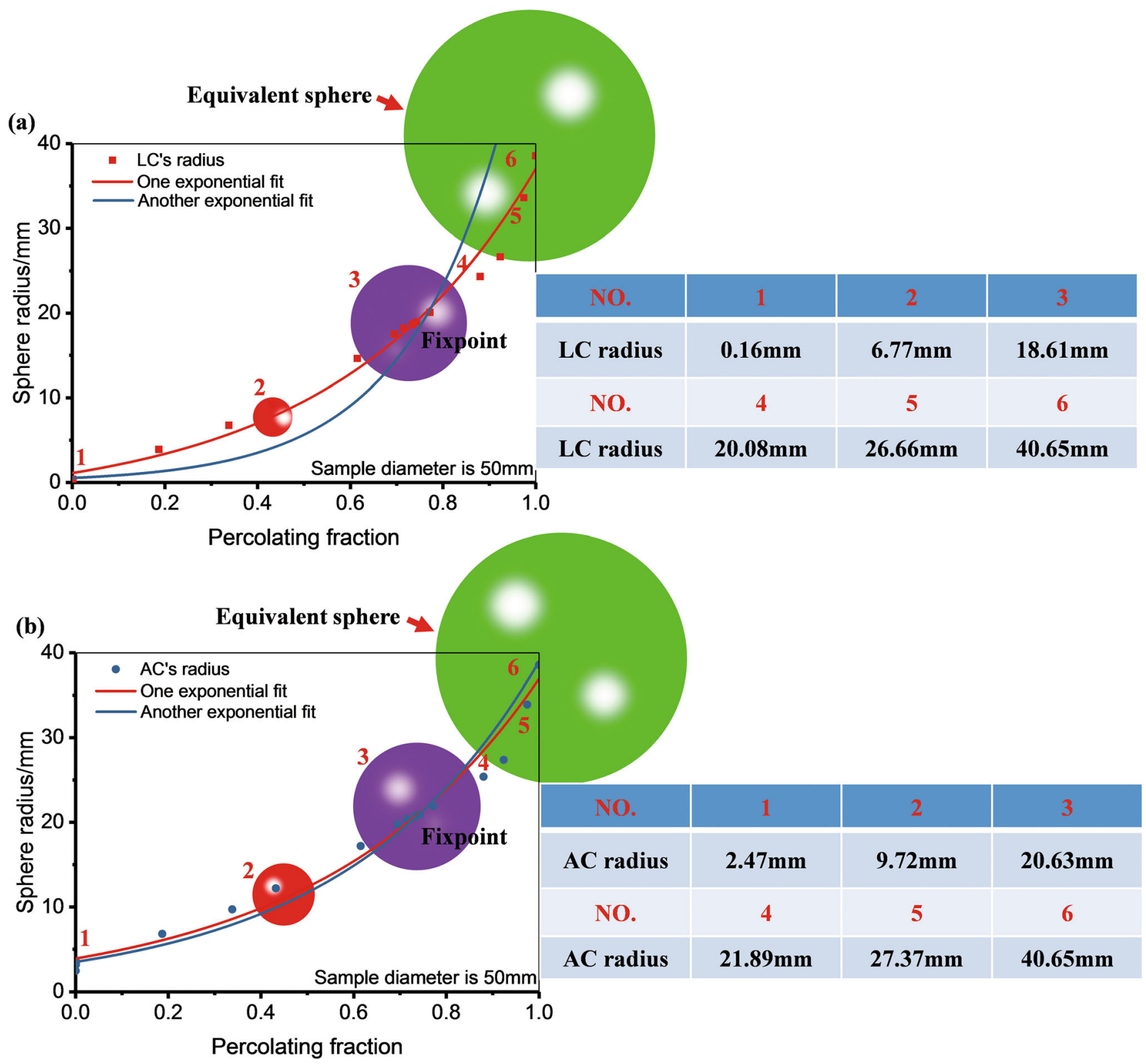

Fig. 6 Equivalent sphere-based isotropic description of $\mathbf{a} \mathrm{LC}$ and $\mathbf{b} \mathrm{AC}$ and the comparison of two fixed points determined by the intersection of two exponential curves

\subsection{Cluster criticality regarding specific surface area}

The specific surface area (SSA) is a key parameter when analyzing the complex geometry of the cube cluster. The SSA contains the outer and inner surfaces of cube cluster because the entire cluster is inside the cylinder. Figure 10 shows the SSA decreasing with increasing PF. The dramatic drop in the total number of cubes seriously reduces the inner area due to connections. As fractures propagate, they induce the continuous generation of $\mathrm{AE}$ signals and correspondingly, the cube cluster enlarges with more surface areas.
Geometrically, the sphere has the smallest SSA of all shapes, and the analysis of cluster criticality regarding isotropy showed the effectiveness of the sphere-based description of dilatancy (Fig. 6). Here, the fracture propagation is mainly accompanied by high SSA. Generally, small $\mathrm{PF}$ indicates that the fracture propagation is preferably observed on the small scale, whereas large PF means that the fracture dilatancy is easily noticed on the large scale. This means that using small cubes to cover the $\mathrm{AE}$ signals is beneficial for observing the fracture propagation. In extreme conditions, such as without any fracture dilatancy, the classical model of a single-link cluster tends to be effective in fracture propagation. However, the 
(a)

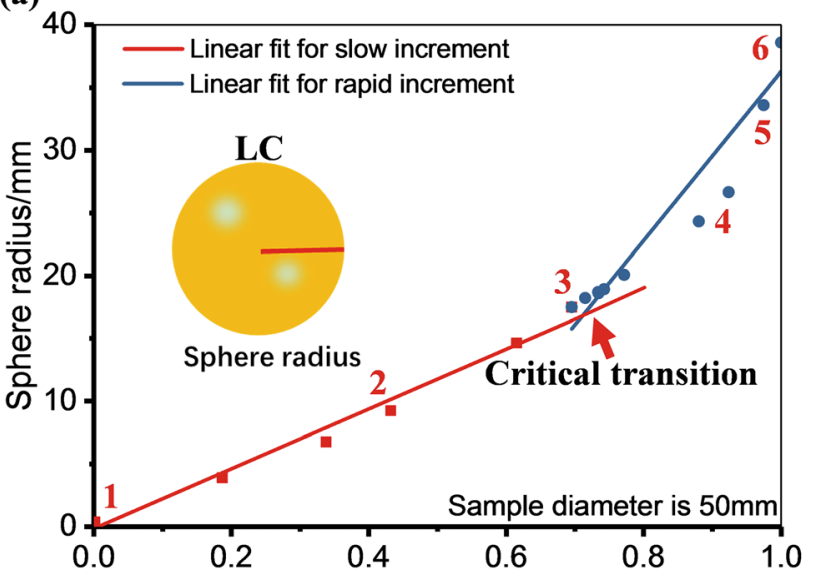

(b)

Percolating fraction

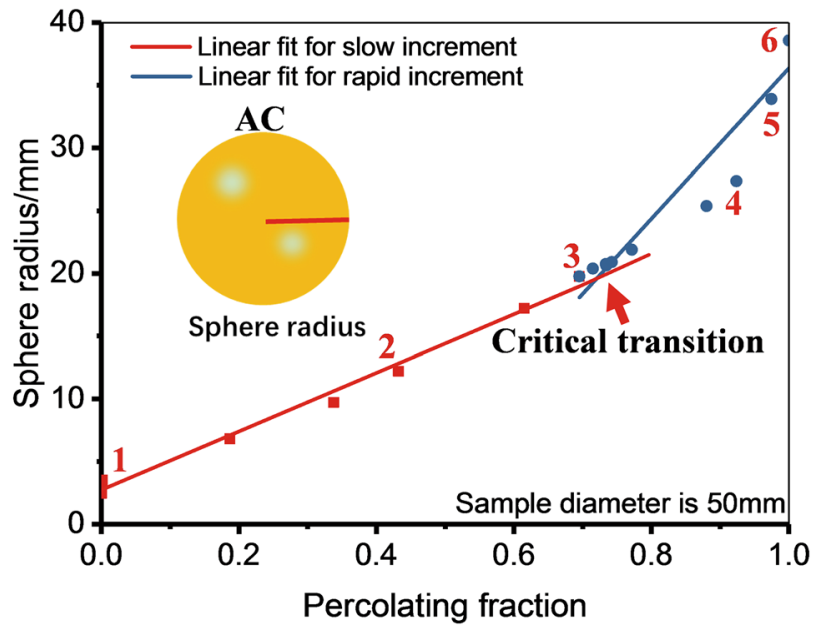

Fig. 7 Determination and comparison of two critical transition points for $\mathbf{a} \mathrm{LC}$ and $\mathbf{b} \mathrm{AC}$ by the intersection of two lines

challenge remains of how to describe dilatancy in cluster modeling, and the key to solving this problem is to use large cubes for the covering. However, using cubes that are too large will also induce the complete attention on the fracture dilatancy ignoring the propagation behavior. Therefore, for a balance between observing propagation and dilatancy, a proper cube-based scale is needed based on the SSA.

Figure 11 shows how the SSA evolves at the six points on the AE curve in Fig. 4. Taking the third point as the transition point, the SSA decreases linearly before that point and increases linearly after that point. Also, the SSA visualized by the ellipsoid is plotted along the fitting lines. The ellipsoid becoming larger corresponds to the rapid growth of the cluster volume. The initial decrease in the SSA indicates the increasing dominance of fracture dilatancy over propagation, after which the increase shows a stronger influence of the SSA on fracture propagation than the dilatancy. Taking each cluster to be a sphere, the corresponding six SSAs are also shown in Fig. 11. Compared with the original SSAs, these new ones are all obviously lower. The ratio of the cluster SSA to that of the equivalent sphere is calculated to evaluate the deviation from isotropic dilatancy, and there is a positive linear correlation between the SSA ratio and the volumetric strain. Indeed, the fracture propagation and opening contribute to the isotropic and anisotropic dilatancy, respectively. However, the fracture propagation obviously has by far the larger influence on the rapid increase of the SSA, and so the criticality regarding the SSA mainly reflects the transition from isotropic to anisotropic dilatancy.

\section{Discussion}

\subsection{Relationship between pore fraction and critical cube length}

In the same way as described previously, another three granite samples were tested under triaxial compression with confining pressures of 5, 20, and $30 \mathrm{MPa}$, respectively. Figure 12 shows the same dilatancy-induced surge of accumulated AE events for these three samples, but with complex cluster evolution. All the cluster geometries and topographies are completely different. By the same bisection of pore fraction and cube size, the cube length of side under 5,20 , and $30 \mathrm{MPa}$ is calculated as $2.00,2.54$, and $2.30 \mathrm{~mm}$, respectively. Considering the critical length of $1.79 \mathrm{~mm}$ at the confining pressure of $10 \mathrm{MPa}$, the critical size shows no obvious dependence on the confining pressure. However, the four cube lengths of 2.00, 1.79, 2.54, and $2.30 \mathrm{~mm}$ show very little difference with the average diameter of three mineral particles in Fig. 1. This suggests strongly that in cube cluster modeling, the best scale for observing the fracture connections is the particle size.

Figure 13 shows a linear relationship between the critical cube length and the LC pore fraction, which in turn shows that cube cluster modeling of an AE signal cloud could be used to predict quantitatively the fraction of a sample that is damaged. Generally, although it is extremely important to have a reliable model for evaluating damage, most attention is paid to extrinsic influences such as the maximum principal stress, the confining pressure, and even the deviatoric stress. The prerequisite for the establishment of such reliable model is that the constraint of confining pressure could inhibit the randomness of fracture propagation. The intrinsic geometric structure is mainly correlated with the spatial distribution of the mineral particles. Without considering the variation of inner structure, the external factor of confining pressure will be ineffective in describing the correlation with intrinsic geometric variables. As mentioned above, it is difficult to establish a certain relationship between pore fraction and confining 

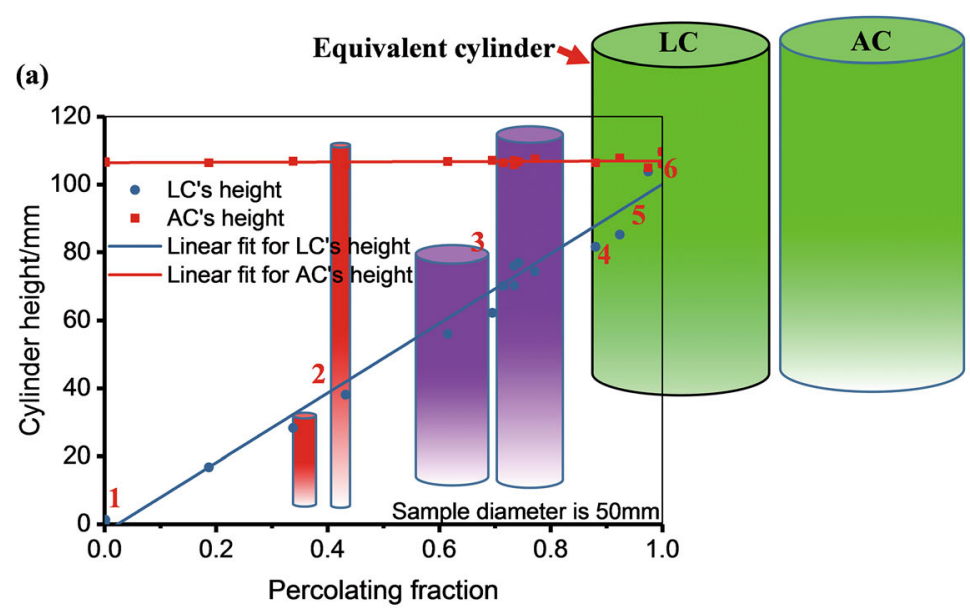

\begin{tabular}{|c|c|c|c|}
\hline No. & 1 & 2 & 3 \\
\hline LC height & $0.40 \mathrm{~mm}$ & $28.32 \mathrm{~mm}$ & $70.32 \mathrm{~mm}$ \\
\hline AC height & $106.72 \mathrm{~mm}$ & $106.86 \mathrm{~mm}$ & $105.82 \mathrm{~mm}$ \\
\hline No. & 4 & 5 & 6 \\
\hline LC height & $74.44 \mathrm{~mm}$ & $85.24 \mathrm{~mm}$ & $106.06 \mathrm{~mm}$ \\
\hline AC height & $107.60 \mathrm{~mm}$ & $107.80 \mathrm{~mm}$ & $107.09 \mathrm{~mm}$ \\
\hline No. & 1 & 2 & 3 \\
\hline LC radius & $0.12 \mathrm{~mm}$ & $3.82 \mathrm{~mm}$ & $11.06 \mathrm{~mm}$ \\
\hline AC radius & $0.43 \mathrm{~mm}$ & $3.39 \mathrm{~mm}$ & $10.52 \mathrm{~mm}$ \\
\hline No. & 4 & 5 & 6 \\
\hline$L C$ radius & $12.04 \mathrm{~mm}$ & $17.21 \mathrm{~mm}$ & $29.06 \mathrm{~mm}$ \\
\hline AC radius & $11.40 \mathrm{~mm}$ & $15.93 \mathrm{~mm}$ & $29.06 \mathrm{~mm}$ \\
\hline
\end{tabular}

(b)

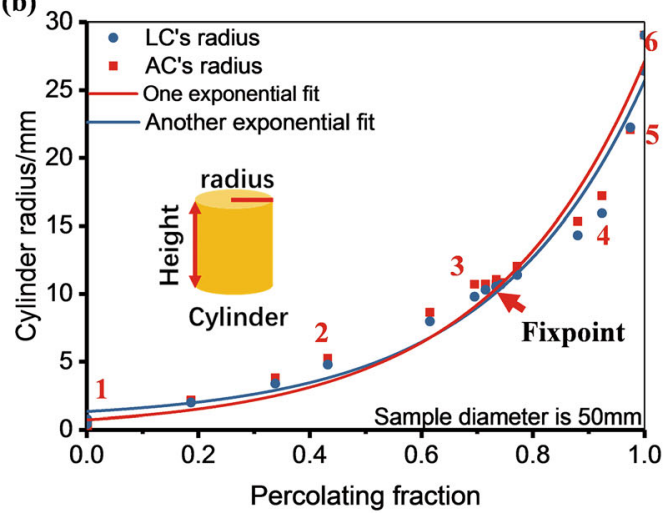

(c)

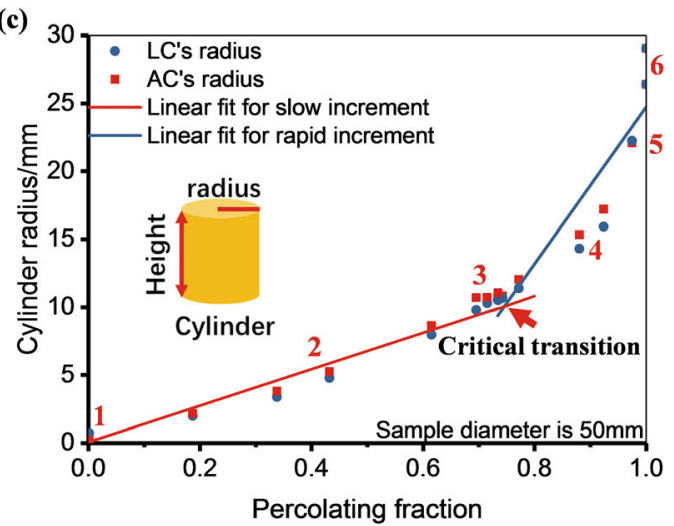

Fig. 8 Equivalent cylinder-based anisotropic description of a LC and AC and the coincidence of $\mathbf{b}$ fixed point and $\mathbf{c}$ critical point dependent on the exponential and linear description

pressure, and the key to solving the problem is a geometric description of the fracture network based on a proper model. Here, the cube cluster modeling of randomly distributed $\mathrm{AE}$ signals provides a direct description of the fracture connections, and the highly linear relationship between the pore fraction and the critical cube length shows a better sensitivity of cube cluster on damage description than the confining pressure.

\subsection{Relationship between confining pressure and multifractality}

Fracture networks often exhibit fractal similarity, a feature that is also used to describe the complex geometry of cube clusters. However, if the geometry becomes extremely complicated, then it cannot be described properly using a single fractal dimension. For a more detailed description, different fractal subsets must be considered for a multiscale observation of the self-similarity, thereby necessitating a multifractal description. The geometric complexity of the cube cluster depends strongly on the stochastic distribution of the $\mathrm{AE}$ scattering signals. By the volume covering method (Zhou et al. 2014), cubes of various scales are used to cover the randomly distributed AE signals, and so the corresponding fractal dimension $d$ is determined by

$d=-\frac{\lg \left(N / N_{0}\right)}{\lg \left(\delta / \delta_{0}\right)}$

where $N_{0}$ is the initial cube number, and $\delta_{0}$ is the initial cube length.

Figure 14 shows plots of the logarithm of the normalized total cube number versus that of the normalized cube length for LC and AC. Considering the AC power-law description, there are four separate linear stages with different fractal dimensions in the corresponding local ranges. The corresponding LC plot deviates considerably from the $\mathrm{AC}$ one when the covering cube length is less than $2.6 \mathrm{~mm}$, whereas they nearly coincide when the covering cube length is greater than $2.6 \mathrm{~mm}$. The point at which they bifurcate corresponds closely to the critical cube length of $1.79 \mathrm{~mm}$. The gradual variation of piecewise-linear coefficients presents the multifractality of AC. However, the branching point divides the corresponding LC curve into two parts: when the cube length of side is less than the 
(a)

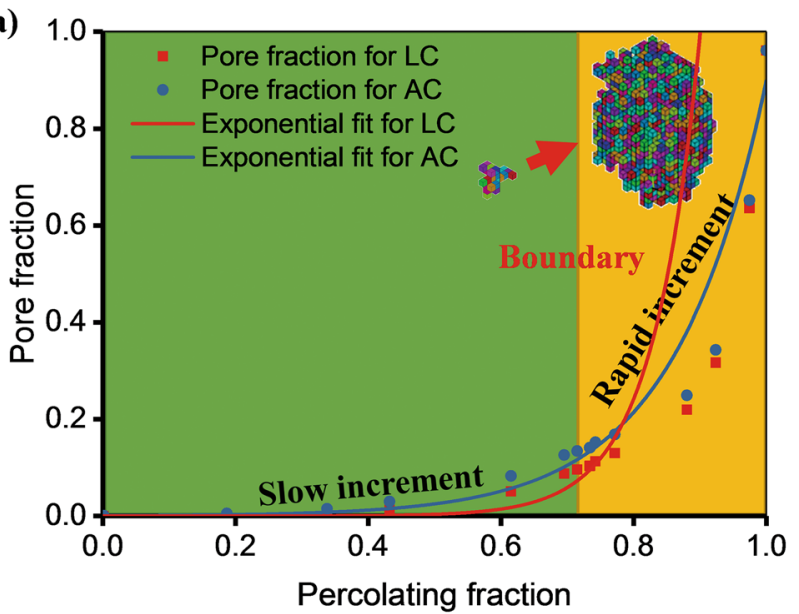

(b)

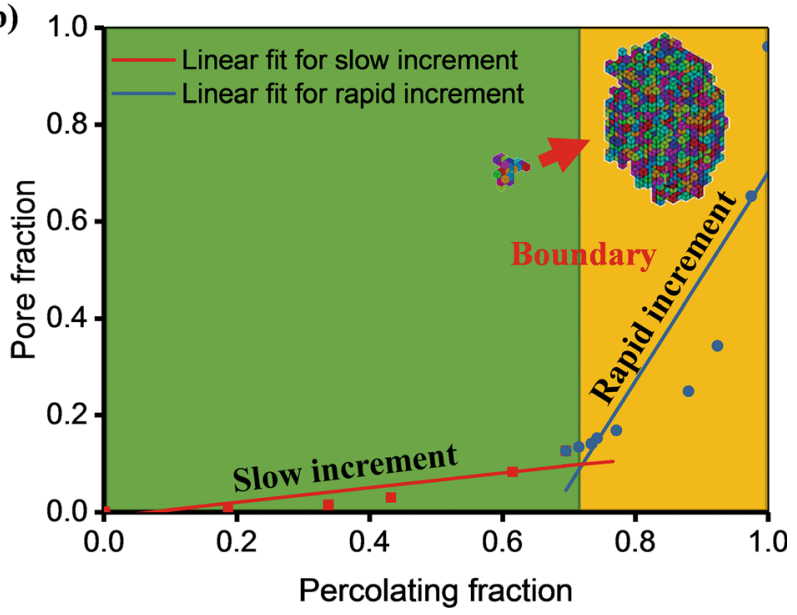

Fig. 9 Relationship between pore fraction and percolation fraction (PF) of LC and AC by a exponential description and $\mathbf{b}$ linear description

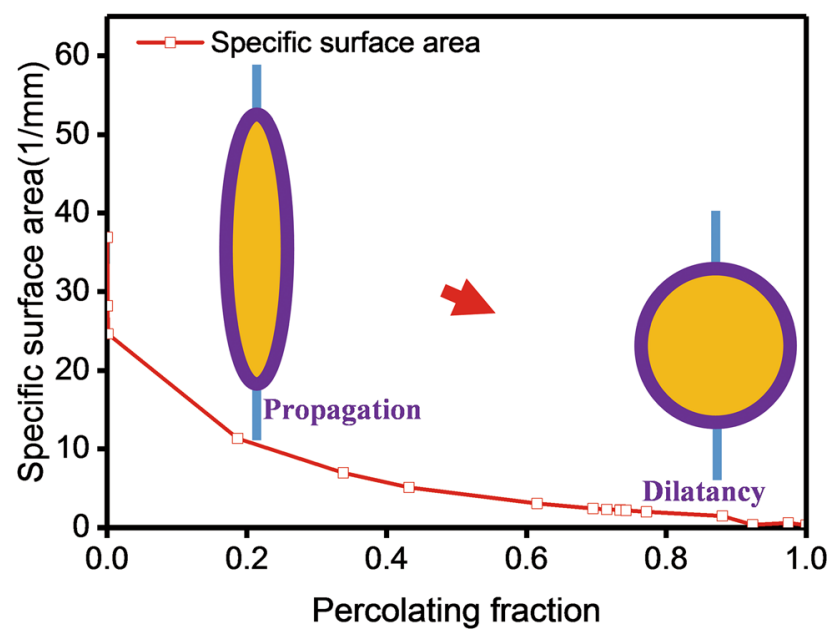

Fig. 10 Relationship between specific surface area (SSA) and PF and its descriptive correlation with fracture propagation and dilatancy

critical length, there is almost no fractal similarity; at and after the branching point, there is obvious multifractality

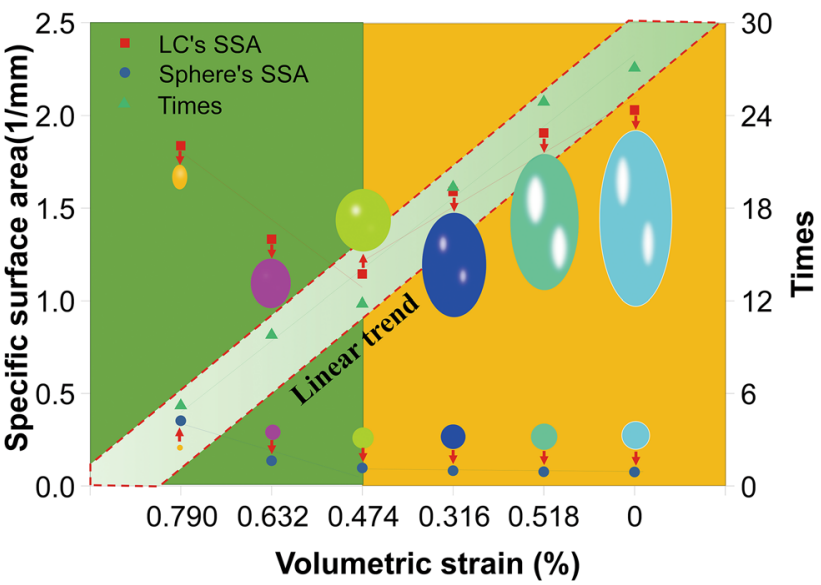

Fig. 11 Relationship between SSA of cube cluster and its equivalent sphere, as well as the evolution of the SSA ratio between the cube cluster and the equivalent sphere

and so the cube cluster (which depends on the critical length) still requires a multifractal description.

In the multifractal description, the singularity $\alpha$ is commonly used to characterize the multifractal spectrum $f(\alpha)$ by

$\alpha=\frac{\lg (\bar{p})}{\lg (\delta)}$

where $\bar{p}$ is the pore fraction taken as the observation of scale invariance. Each singularity $\alpha$ matches the total cube number $N(\alpha)$ uniquely, so the spectrum $f(\alpha)$ is calculated as

$f(\alpha)=-\frac{\lg (N(\alpha))}{\lg (\delta)}$

Chhabra and Jensen (1989) proposed a method for calculating a multifractal spectrum by introducing the discrete form of the generalized fractal dimension $D(q)$, namely

$D(q) \alpha=\frac{1}{q-1} \lim _{l \rightarrow 0} \lg \sum_{k}^{n} P_{k}^{q}(\delta) / \lg \delta$

where $q$ is the weight of multifractal spectrum and $k=$ $1,2, \cdots, n$ is the number of subsets. By means of a Legendre transformation, the descriptive system $\alpha \sim f(\alpha)$ is equivalent to the system $q \sim D(q)$. The singularity then becomes

$\alpha(q)=\frac{\mathrm{d}}{\mathrm{d} q}[(q-1) D(q)]$

and the multifractal spectrum becomes

$f(q)=q \alpha(q)-(q-1) D(q)$

By constructing a series of normalized parameters $\mu_{k}$ as the measured family of 
(a)

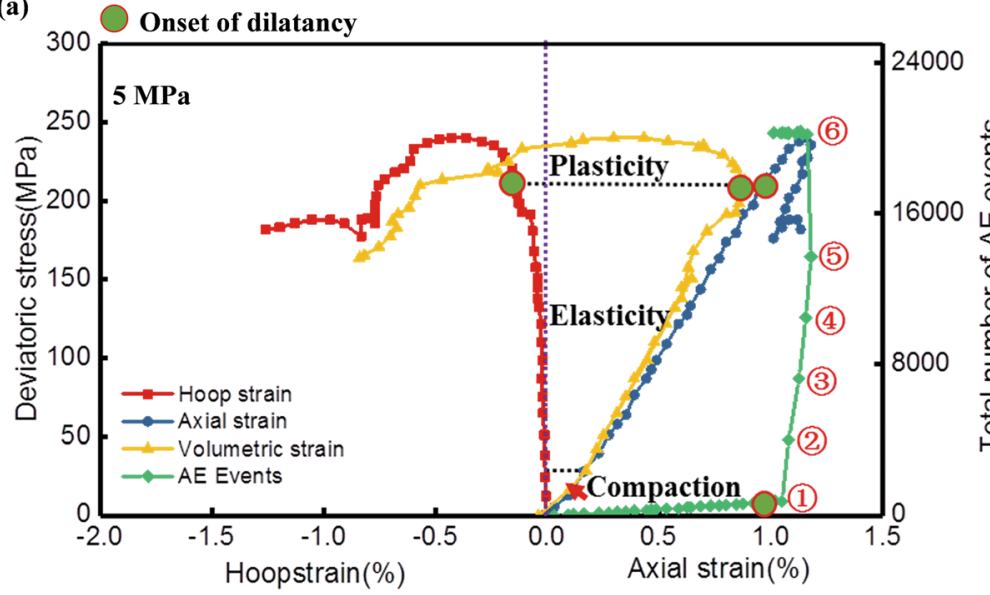

(b)

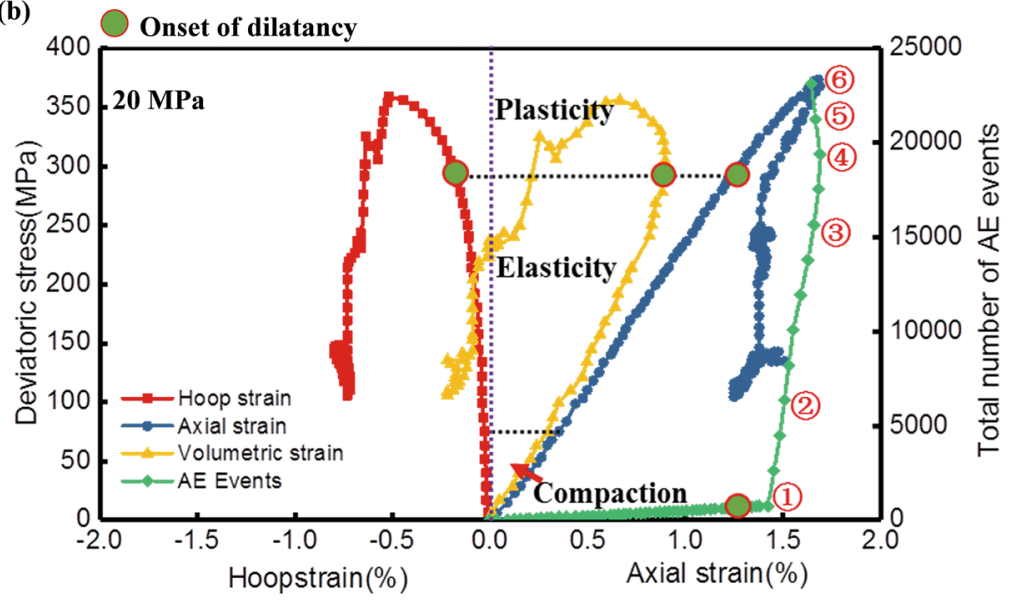

AC

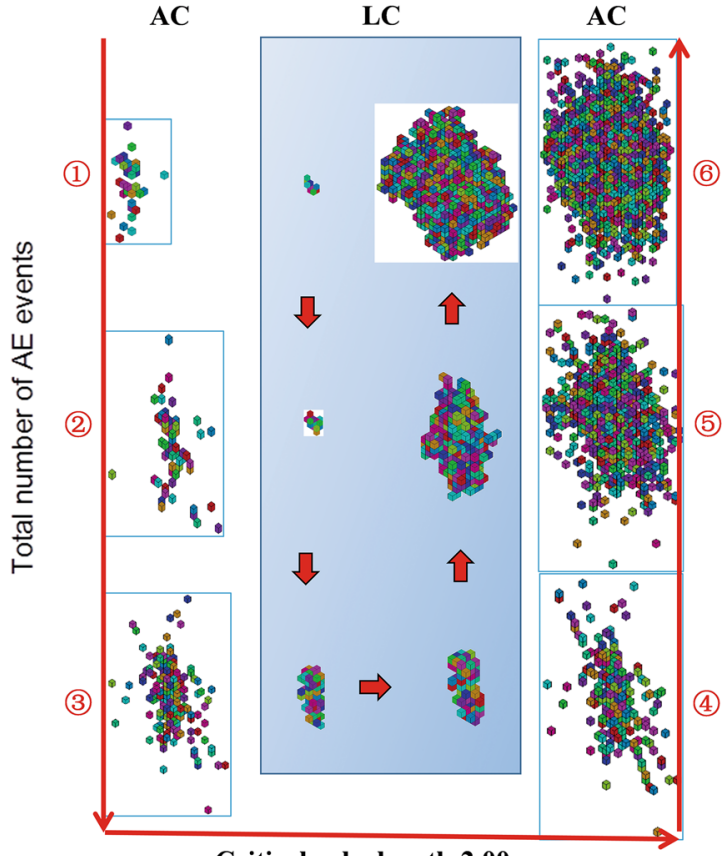

Critical cube length: $2.00 \mathrm{~mm}$.

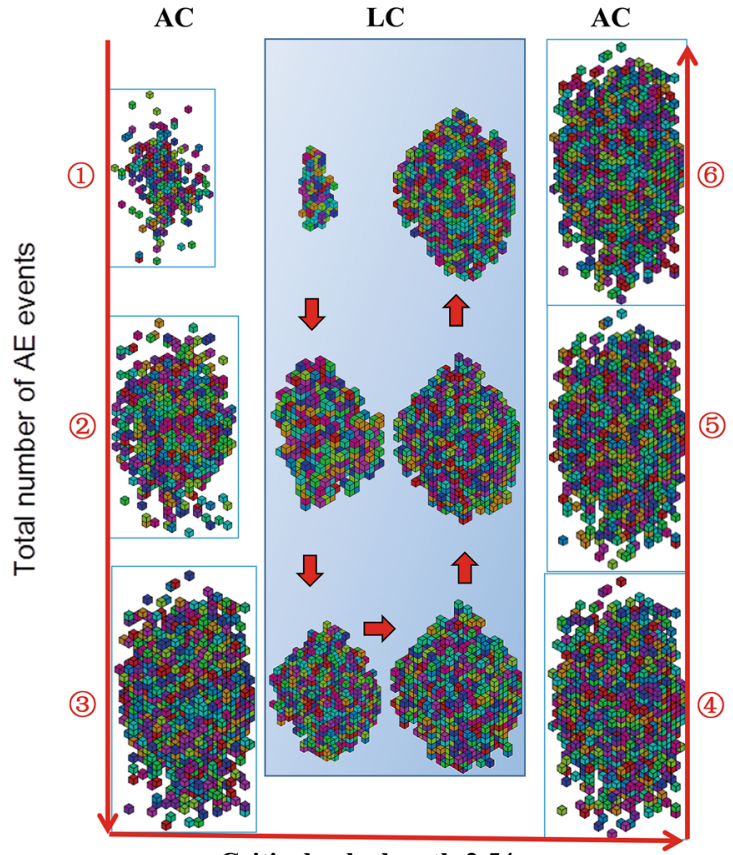

Critical cube length: $2.54 \mathrm{~mm}$.

Fig. 12 Complete stress-strain curves and cube cluster evolution of LC and AC for three samples under confining pressures of a $5 \mathrm{MPa}$, b $20 \mathrm{MPa}$, and c $30 \mathrm{MPa}$

$\mu_{k}(q, \delta)=\left[P_{k}^{q}(\delta)\right] / \sum_{k}^{n} P_{k}^{q}(\delta)$

and substituting Eq. (13) into Eqs. (11) and (12), we have

$\alpha(q)=\lim _{\delta \rightarrow 0}\left[\sum_{k}^{n} \mu_{k}(q, \delta) P_{k}(\delta) / \lg \delta\right]$

and $f(q)=\lim _{\delta \rightarrow 0}\left[\sum_{k}^{n} \mu_{k}(q, \delta) \lg \mu_{k}(q, \delta) / \lg \delta\right]$

Figure 15 shows the evolution of the LC multifractal spectrum at the six states for the four samples under confining pressures of $5,10,20$, and $30 \mathrm{MPa}$, respectively. To investigate the multifractal spectrum, the difference descriptors $\Delta \alpha=\alpha_{\max }-\alpha_{\min }$ and $\Delta f=f\left(\alpha_{\min }\right)-f\left(\alpha_{\max }\right)$ 
(c)

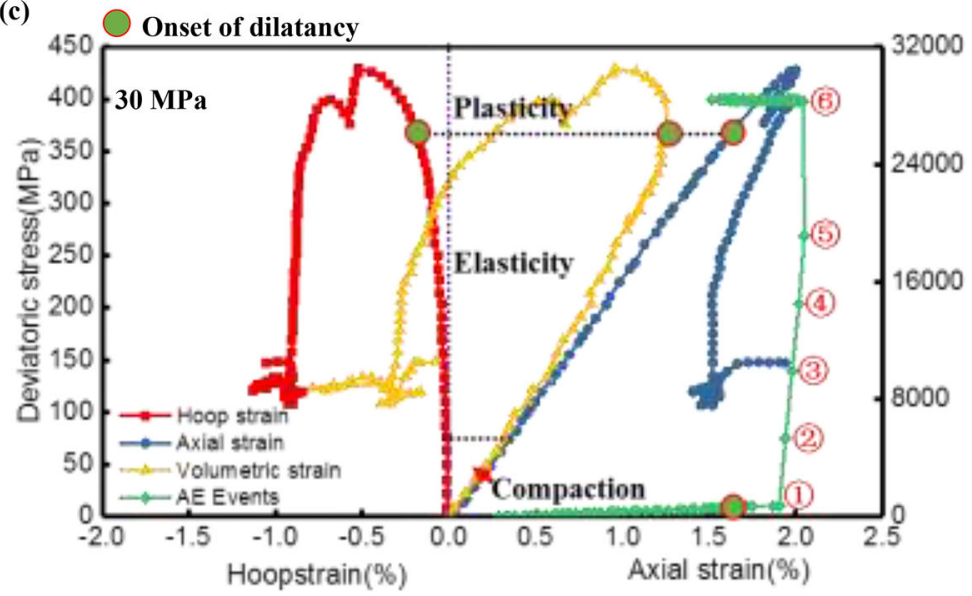

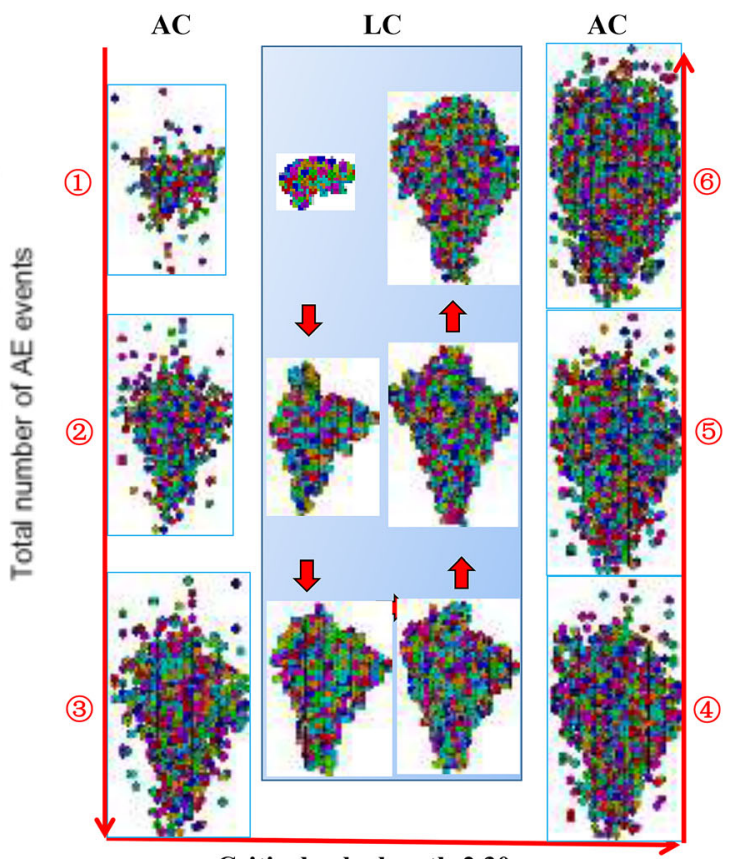

Critical cube length:2.30mm

Fig. 12 continued

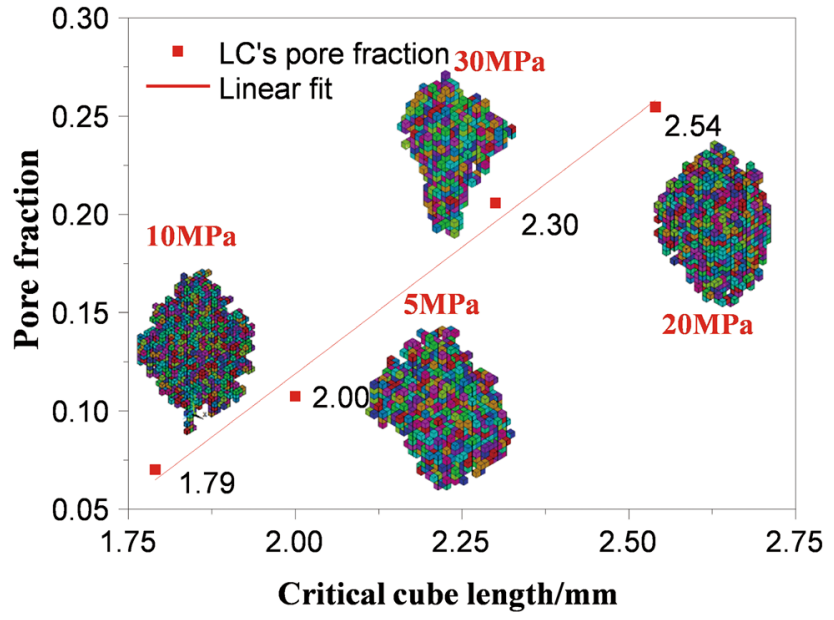

Fig. 13 Linear correlation between pore fraction and critical cube length

are generally used. The first descriptor, $\Delta \alpha$, indicates the openness of the spectrum curve; the larger $\Delta \alpha$, the greater the distribution range of the pore fraction. For the other descriptor, $\Delta f$, usually the larger it is, the greater the difference between the maximum and minimum pore fraction. Nearly all the spectra have $\Delta f>0$, which means that the number of peaks is always larger than that of the accumulated valleys and that is because some constant peaks continuously distribute.

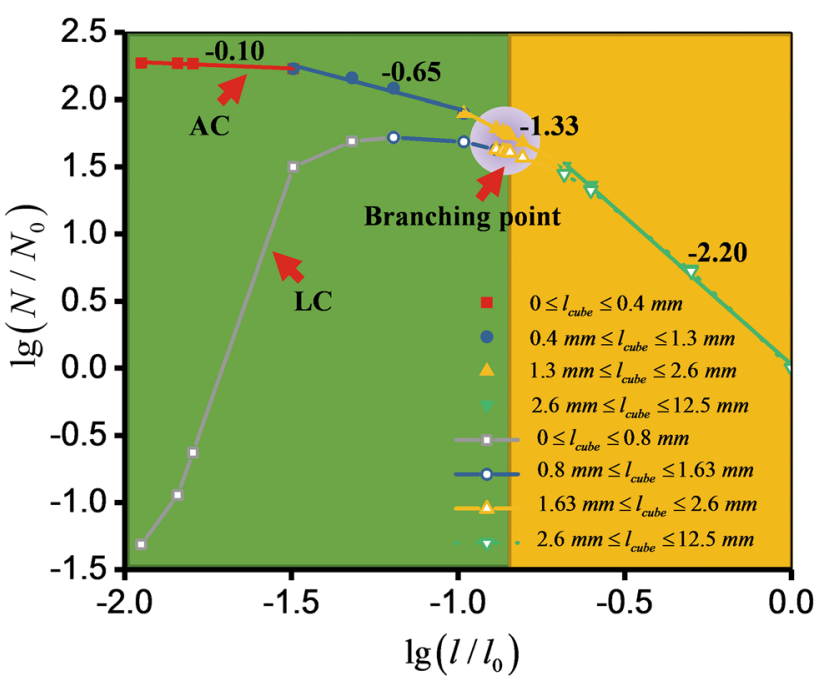

Fig. 14 Plots of logarithm of normalized covering-cube number versus logarithm of normalized cube length for LC and AC to reveal the multifractality

Finally, Fig. 16 shows the piecewise linear relationship between the singularity increment $\Delta \alpha$ and the confining pressure. Clearly, $\Delta \alpha$ decreases with increasing confining pressure, especially at low values of the latter. Decreasing $\Delta \alpha$ means that the pore fraction fluctuates less, which is caused mainly by the confining constraint under high pressure. In other words, the layer clusters at various positions have similar cube numbers. In general, the pore 
(a)
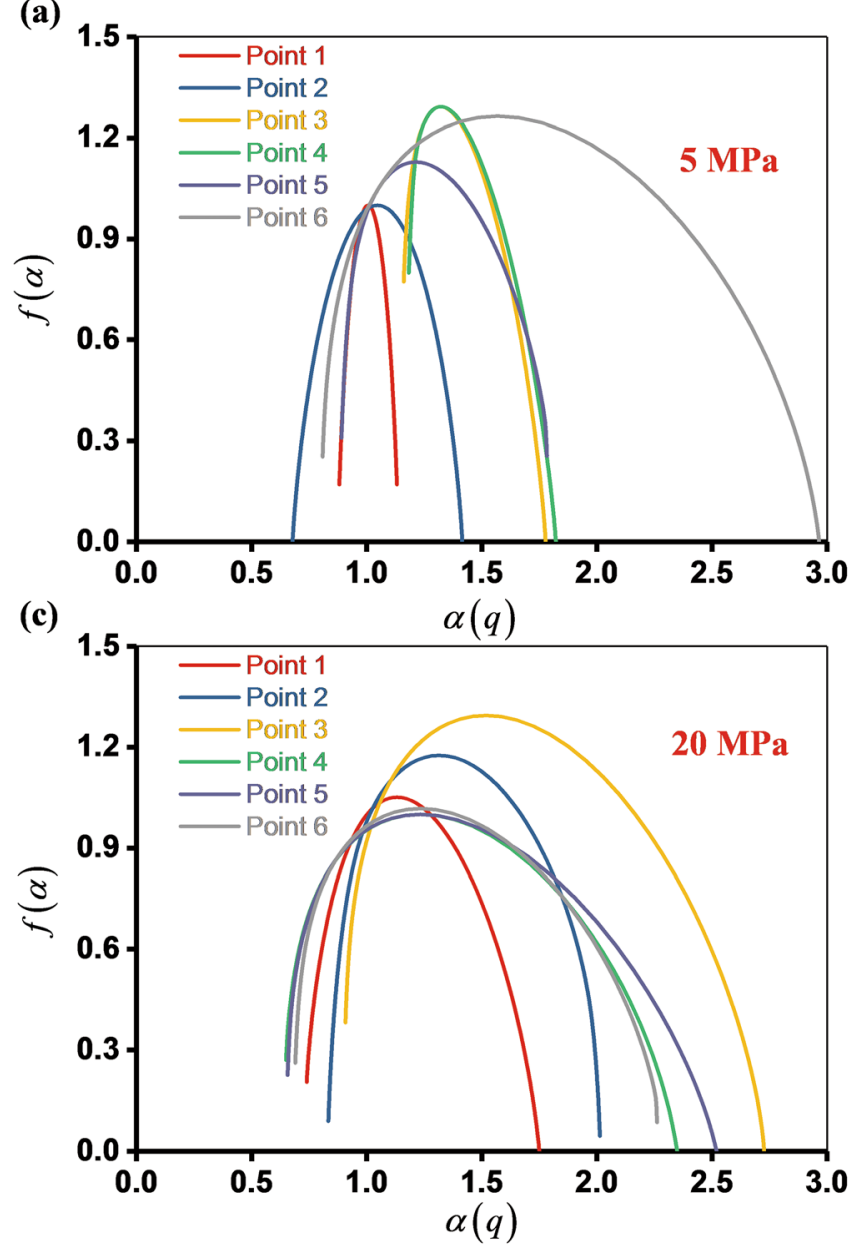

(b)

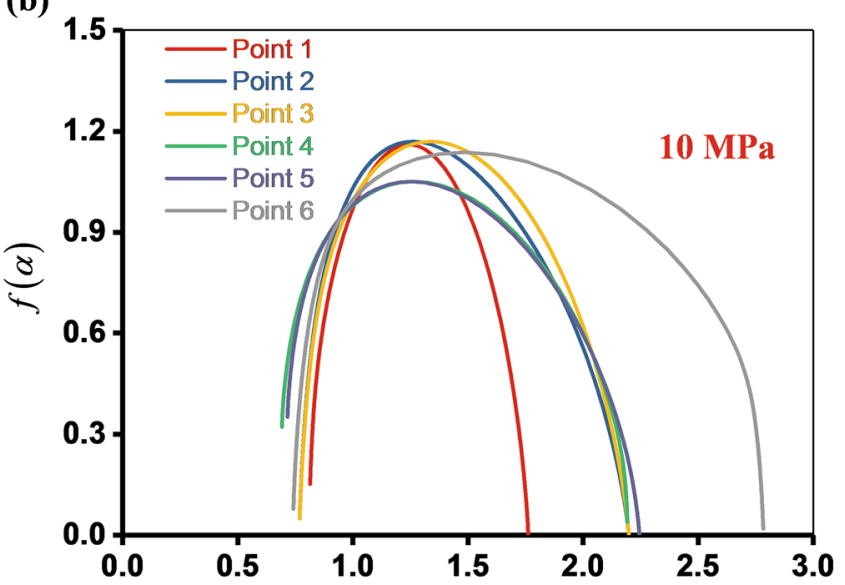

(d)

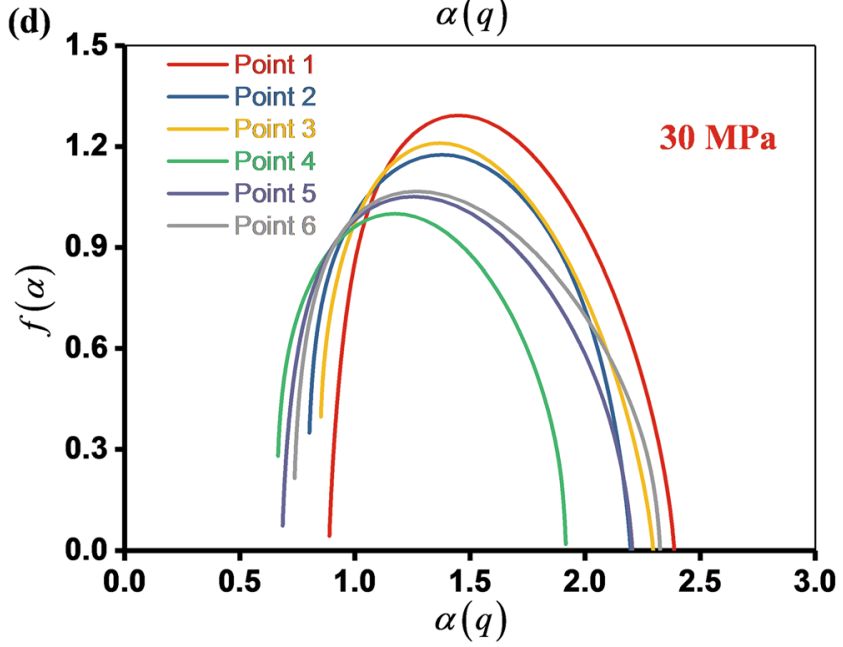

Fig. 15 Multifractal spectrum evolution of LC under a $5 \mathrm{MPa}$, b $10 \mathrm{MPa}$, c $20 \mathrm{MPa}$, and d $30 \mathrm{MPa}$

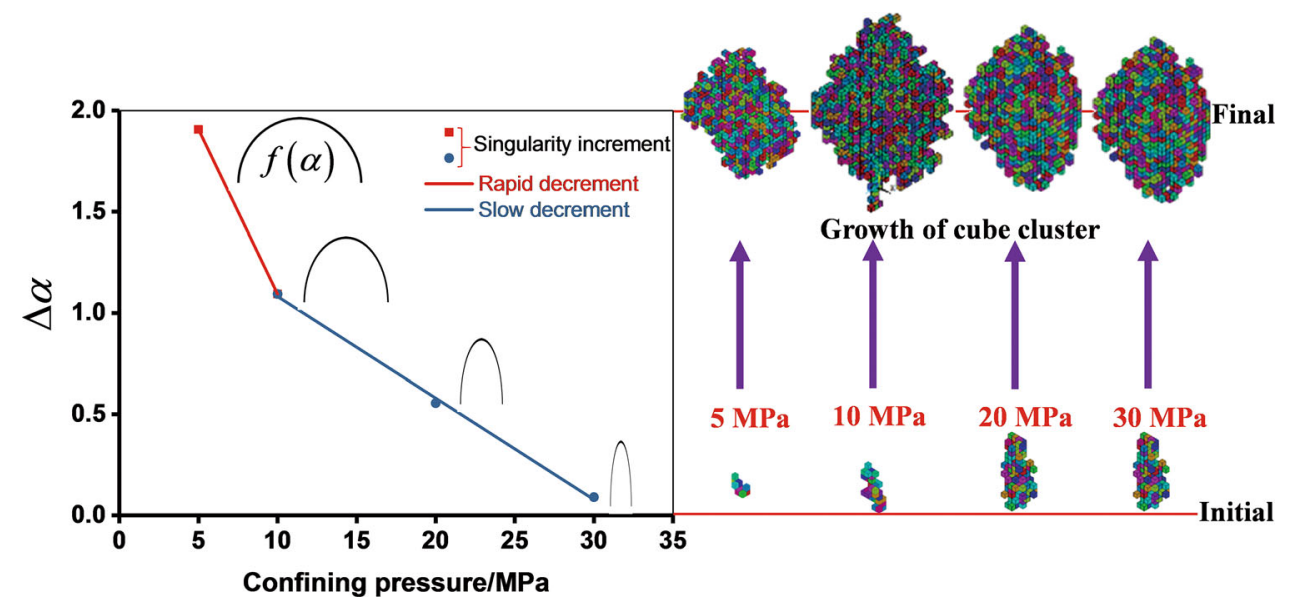

Fig. 16 Relationship between singularity increment and confining pressure 
fraction given by cube cluster modeling of randomly distributed AE signals is correlated strongly with the critical cube length. However, in the multifractal description, the distribution of the pore fraction is influenced mainly by the confining pressure.

\section{Conclusions}

In geotechnical engineering, $\mathrm{AE}$ monitoring technology is highly promising for investigating the invisible failure process in rock. However, the lack of proper modeling of the spatial correlation of randomly distributed AE signals hinders its further development to establish a predictive correlation between the AE signal cloud and the triaxial compression behavior. With the aim of providing a new way to describe the spatial correlation, the cube cluster model was proposed and analyzed herein, and the main conclusions are as follows.

Considering the criticality of connections in the fracture network, the cube cluster model of the AE scattering signals was established effectively. Intrinsically in topology, the failure of rock under triaxial compression is an evolution of fracture connectivity. The completeness of the main fracture network is defined as a critical state of connection that corresponds to the increasing-to-decreasing transition of the deviatoric stress. The criticality of the cube cluster was verified also by the other three geometric descriptors of anisotropy, pore fraction, and SSA. The criticality of the topographic connection directly induces the geometric criticality of these three descriptors.

The cube cluster model was built on the short-range correlation of the AE scattering signals. Unlike the scattering description of a randomly distributed AE signal cloud, the cube cluster model focuses on spatial correlation instead of spatial distribution, and it originates from the scientific observation of dependence of the post-generation of AE signals on pre-ones. Unlike the traditional modeling in which a single link connects any two isolated points directly, the cube cluster model is based on the local-scale observation of the interaction of adjacent signals in the same critical cube. Having built the short-range correlation in a cube, the long-range connection of the fracture network could be realized by the surface-to-surface contact.

The pore fraction considering the locally damaged zones around the fractures is influenced by the critical cube length and the confining pressure. There is a positive linear correlation between the pore fraction and the critical cube length, and based on the multifractal spectrum of the porefraction distribution there is a negative linear correlation between the singularity increment and the confining pressure.
Funding This study was sponsored by the National Natural Science Foundation of China (No. 51504257), the State Key Research Development Program of China (No. 2016YFC0600704), the Fund of Yueqi Outstanding Scholars (No. 2018B051616), and the Open Fund of the State Key Laboratory of Coal Mine Disaster Dynamics and Control (No. 2011DA105287- FW201604).

Data availability The data used to support the findings of this study are available from the corresponding author upon reasonable request.

\section{Compliance with ethical standards}

Conflict of interest The authors declare no conflict of interest.

Open Access This article is licensed under a Creative Commons Attribution 4.0 International License, which permits use, sharing, adaptation, distribution and reproduction in any medium or format, as long as you give appropriate credit to the original author(s) and the source, provide a link to the Creative Commons licence, and indicate if changes were made. The images or other third party material in this article are included in the article's Creative Commons licence, unless indicated otherwise in a credit line to the material. If material is not included in the article's Creative Commons licence and your intended use is not permitted by statutory regulation or exceeds the permitted use, you will need to obtain permission directly from the copyright holder. To view a copy of this licence, visit http://creativecommons. org/licenses/by/4.0/.

\section{References}

Aharony A, Stauffer D (2014) Introduction to percolation theory: revised, 2nd edn. CRC Press, Boca Raton

Al-Futaisi A, Patzek TW (2003) Extension of Hoshen-Kopelman algorithm to non-lattice environments. Phys A 321:665-678

Alkan H (2009) Percolation model for dilatancy-induced permeability of the excavation damaged zone in rock salt. Int J Rock Mech Min 46:716-724

Alkan H, Cinar Y, Pusch G (2007) Rock salt dilatancy boundary from combined acoustic emission and triaxial compression tests. Int J Rock Mech Min 44:108-119

Bebbington M, Vere-Jones D, Zheng X (1990) Percolation theory: a model for rock fracture. Geophys J Int 100:215-220

Broadbent SR, Hammersley JM (1957) Percolation processes: I. Crystals and mazes. In: Mathematical proceedings of the Cambridge philosophical society. Cambridge University Press 53:629-641

Cai M, Morioka H, Kaiser PK, Tasaka Y, Kurose H, Minami M, Maejima T (2007) Back-analysis of rock mass strength parameters using $\mathrm{AE}$ monitoring data. Int $\mathrm{J}$ Rock Mech Min 44:538-549

Chang SH, Lee CI (2004) Estimation of cracking and damage mechanisms in rock under triaxial compression by moment tensor analysis of acoustic emission. Int J Rock Mech Min 41:1069-1086

Chelidze TL (1982) Percolation and fracture. Phys Earth Planet Inter 28:93-101

Chelidze TL (1986) Percolation theory as a tool for imitation of fracture process in rocks. Pure Appl Geophys 124:731-748

Chevy J, Fressengeas C, Lebyodkin M, Taupin V, Bastie P, Duval P (2010) Characterizing short-range vs. long-range spatial correlations in dislocation distributions. Acta Mater 58:1837-1849

Chhabra A, Jensen RV (1989) Direct determination of the $f(\alpha)$ singularity spectrum. Phys Rev Lett 62:1327-1330 
Girard L, Weiss J, Amitrano D (2012) Damage-cluster distributions and size effect on strength in compressive failure. Phys Rev Lett 108(22):1-5

Goodman RE, Blake W (1966) Rock noise in landslides and slope failures. Highway Res Rec 119:50-60

Grosse CU, Ohtsu M (2008) Acoustic emission testing. Springer, Heidelberg

Guo T, Zhang S, Ge H, Wang X, Lei X, Xiao B (2015) A new method for evaluation of fracture network formation capacity of rock. Fuel 140:778-787

Hardy HR (1972) Application of acoustic emission techniques to rock mechanics research. ASTM Spec Tech Publ 1972:41-83

Hestir K, Long J (1990) Analytical expressions for the permeability of random two-dimensional Poisson fracture networks based on regular lattice percolation and equivalent media theories. J Geophys Res: Solid Earth 95:21565-21581

Hirata T, Satoh T, Ito K (1987) Fractal structure of spatial distribution of microfracturing in rock. Geophys J Int 90:369-374

Hohl A, Griffith AD, Eppes MC, Delmelle E (2018) Computationally enabled 4D visualizations facilitate the detection of rock fracture patterns from acoustic emissions. Rock Mech Rock Eng 2018:1-14

Hoshen J, Berry MW, Minser KS (1997) Percolation and cluster structure parameters: the enhanced Hoshen-Kopelman algorithm. Phys Rev E 56:1455

Hsu NN, Simmons JA, Hardy SC (1978) Approach to acoustic emission signal analysis-theory and experiment. In: Proceedings of the ARPA/AFML review of progress in quantitative NDE 166-169

Hunt A, Ewing R, Ghanbarian B (2014) Percolation theory for flow in porous media. Springer, New York

Jarvis N, Larsbo M, Koestel J (2017) Connectivity and percolation of structural pore networks in a cultivated silt loam soil quantified by X-ray tomography. Geoderma 287:71-79

Kaiser J (1950) Investigation of acoustic emission in tensile testing. Technische Universität München, Germany

Kusunose K, Lei X, Nishizawa O, Satoh T (1991) Effect of grain size on fractal structure of acoustic emission hypocenter distribution in granitic rock. Phys Earth Planet Inter 67:194-199

Lei X, Kusunose K, Rao MVMS, Nishizawa O, Satoh T (2000) Quasi-static fault growth and cracking in homogeneous brittle rock under triaxial compression using acoustic emission monitoring. J Geophys Res: Solid Earth 105:6127-6139

Li HG, Li H (2017) Mechanical properties and acoustic emission characteristics of thick hard roof sandstone in Shendong coal field. Int J Coal Sci Technol 4(2):147-158

Lockner D (1993) The role of acoustic emission in the study of rock fracture. Int J Rock Mech Min 30:883-899

López-Comino JA, Cesca S, Heimann S, Grigoli F, Milkereit C, Dahm T, Zang A (2017) Characterization of hydraulic fractures growth during the Äspö Hard Rock Laboratory experiment (Sweden). Rock Mech Rock Eng 50:2985-3001

Manthei G (2005) Characterization of acoustic emission sources in a rock salt specimen under triaxial compression. Bull Seismol Soc Am 95:1674-1700

Meng Q, Zhang M, Han L, Pu H, Nie T (2016) Effects of acoustic emission and energy evolution of rock specimens under the uniaxial cyclic loading and unloading compression. Rock Mech Rock Eng 49:3873-3886

Pape H, Clauser C, Iffland J (1999) Permeability prediction based on fractal pore-space geometry. Geophysics 64:1447-1460

Rodríguez P, Arab PB, Celestino TB (2016) Characterization of rock cracking patterns in diametral compression tests by acoustic emission and petrographic analysis. Int $\mathbf{J}$ Rock Mech Min 83:73-85

Sakhaee-Pour A, Agrawal A (2018) Integrating acoustic emission into percolation theory to predict permeability enhancement. J Petrol Sci Eng 160:152-159

Scruby CB (1987) An introduction to acoustic emission. J Phys E 20:946-953

Shante VKS, Kirkpatrick S (1971) An introduction to percolation theory. Adv Phys 20:325-357

Stanchits S, Burghardt J, Surdi A (2015) Hydraulic fracturing of heterogeneous rock monitored by acoustic emission. Rock Mech Rock Eng 48:2513-2527

Sugiyama T, Promentilla MAB, Hitomi T, Takeda N (2010) Application of synchrotron microtomography for pore structure characterization of deteriorated cementitious materials due to leaching. Cement Concr Res 40:1265-1270

Tang CA, Liu H, Lee PKK, Tsui Y, Tham L (2000) Numerical studies of the influence of microstructure on rock failure in uniaxial compression-part I: effect of heterogeneity. Int J Rock Mech Min 37:555-569

Vilhelm J, Rudajev V, Lokajíček T, Veverka J (2008) Correlation analysis of the ultrasonic emission from loaded rock samplesthe study of interaction of microcracking nucleation centres. Rock Mech Rock Eng 41(5):695-714

Wang J, Chen L, Su R, Zhao X (2018) The Beishan underground research laboratory for geological disposal of high-level radioactive waste in China: planning, site selection, site characterization and insitutests. J Rock Mech Geotech Eng 10:411-435

Xie HP, Liu JF, Ju Y, Li J, Xie LZ (2011) Fractal property of spatial distribution of acoustic emissions during the failure process of bedded rock salt. Int J Rock Mech Min 48:1344-1351

Xue DJ, Zhou J, Liu YT, Zhang SS (2018) A strain-based percolation model and triaxial tests to investigate the evolution of permeability and critical dilatancy behavior of coal. Processes 6(8):127

Xue DJ, Zhou J, Liu YT, Lie Gao (2020) On the excavation-induced stress drop in damaged coal considering a coupled yield and failure criterion. Int J Coal Sci Technol 7(1):58-67

Yang SQ, Jing HW, Wang SY (2012) Experimental investigation on the strength, deformability, failure behavior and acoustic emission locations of red sandstone under triaxial compression. Rock Mech Rock Eng 45:583-606

Yuan RF, Bowen Shi (2018) Acoustic emission activity in directly tensile test on marble specimens and its tensile damage constitutive model. Int J Coal Sci Technol 5(3):295-304

Zhao XG, Cai M, Wang J, Ma LK (2013) Damage stress and acoustic emission characteristics of the Beishan granite. Int J Rock Mech Min 64:258-269

Zhou HW, Xue DJ, Jiang DY (2014) On fractal dimension of a fracture surface by volume covering method. Surf Rev Lett 21:1-11 